\title{
Hamartomatosis biliar en una lactante con colitis alérgica. Revisión a propósito de un caso
}

\author{
I. Carabaño Aguado ${ }^{a}$, M. Herrero Álvarez ${ }^{a}$, J. Sánchez Hernández ${ }^{b}$ E. La Orden Izquierdo ${ }^{c}$
}

Publicado en Internet: 9-septiembre-2013

Iván Carabaño Aguado: carabano1975@hotmail.com
aUnidad de Gastroenterología y Nutrición. Servicio de Pediatría. Hospital Rey Juan Carlos.
Móstoles, Madrid. España • 'bServicio de Radiología. Hospital Rey Juan Carlos. Móstoles, Madrid. España
• 'Unidad de Gastroenterología y Nutrición. Servicio de Pediatría. Hospital Infanta Elena.
Valdemoro, Madrid. España.

La hamartomatosis biliar múltiple (HBM) aparece en el 0,9\% de los niños. Su origen está en una disembriogénesis. Los hamartomas de la HBM tienen un color blanco-grisáceo, y pueden estar localizados en la región subcapsular o dispersos por el parénquima. Su tamaño suele ser inferior a $3 \mathrm{~mm}$. Clínicamente, la HBM sigue un curso asintomático. El diagnóstico de la HBM requiere inicialmente una prueba de imagen (ecografía, tomografía computarizada o resonancia magnética). Desde el punto de vista histológico, cada hamartoma consta de varios grupos de conductos biliares intrahepáticos dilatados en un estroma fibrilar de colágeno denso que puede hialinizarse y/o calcificarse. La HBM no precisa tratamiento, dado su bajo potencial de malignización.

Palabras clave:

- Hamartomatosis - Hígado

\section{Biliary hamarthomatosis in an infant with alergic colitis. A case report}

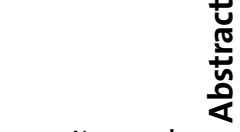

Key words:

- Hamartomatosis

- Liver
Biliary hamartomatosis $(\mathrm{BH})$ occurs in $0.9 \%$ of children. Its origin is dysembriogenesis. Hamartomas of this disease show grey-white color, and can be located in subcapsular region or scattered at the parenchyma. Its size is usually less than $3 \mathrm{~mm}$. Clinically, BH follows an asymptomatic course. The diagnosis of HBM initially requires an imaging test (ultrasound, CT or MRI). Histologically, each hamartoma has several groups of dilated intrahepatic bile ducts in a stroma that may be hyalinazed and/or calcified. $\mathrm{BH}$ do not require treatment, because its malignant potential is slow.

\section{CASO CLÍNICO}

Lactante de un mes y medio de vida, alimentada con leche materna a demanda, derivada a las consultas externas de Gastroenterología de nuestro hospital por regurgitaciones frecuentes, cambio en el hábito intestinal (emisión de diez deposiciones diarias blandas, con moco), rectorragia intermitente y prolapso rectal. El estudio preliminar, que incluyó detección en heces de virus y bacterias, así como hemograma, bioquímica y estudio de coagulación, fue normal. Tres días antes de ser vista en las consultas externas, por progresión del número de vómitos, se le pidió desde Urgencias una ecografía abdominal, en la que se descartó la existencia de signos de estenosis hipertrófica de píloro; páncreas, bazo, riñones y vejiga fueron normales. El hígado mostró un tamaño normal y un borde liso, pero en él se identificaron múltiples imágenes puntiformes hiperecóicas (Fig. 1), alguna de ellas

Cómo citar este artículo: Carabaño Aguado I, Herrero Álvarez M, Sánchez Hernández J, La Orden Izquierdo E. Hamartomatosis biliar en una lactante con colitis alérgica. Revisión a propósito de un caso. Rev Pediatr Aten Primaria. 2013;15:254.e111-e114. 


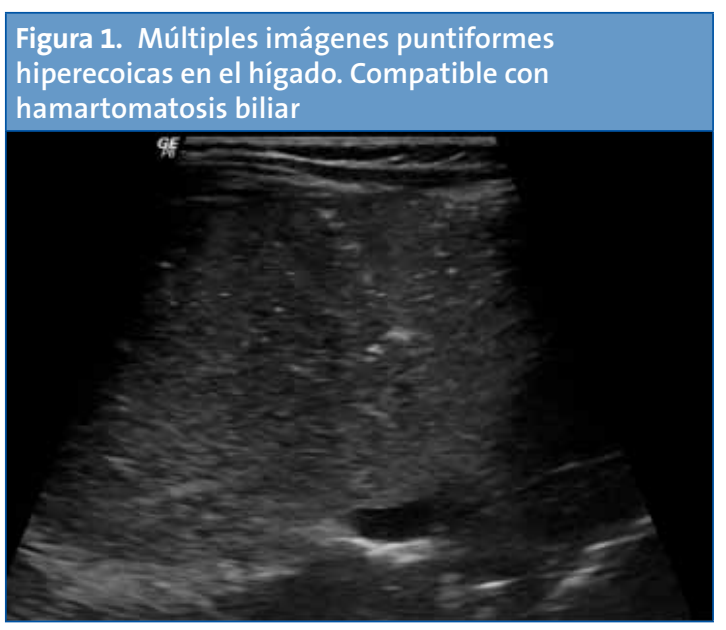

con artefacto en cola de cometa (Fig. 2), en relación con hamartomatosis biliar.

La paciente fue finalmente diagnosticada de colitis por alergia a las proteínas de leche de vaca no mediada por IgE. Se documentó histopatológicamente un rico infiltrado eosinofílico en la lámina propia y la submucosa rectal (más de 60 eosinófilos por 10 campos de gran aumento). La exclusión de lácteos por parte de la madre no resultó exitosa clínicamente hablando, por lo que se tuvo que recurrir a la administración de fórmula láctea hidrolizada. En la actualidad, la lactante cuenta con ocho meses de vida y se encuentra asintomática.

\section{DISCUSIÓN}

La hamartomatosis biliar múltiple (HBM) o enfermedad de los complejos de von Meyenburg aparece en el 0,9\% de los niños y en el 5,6\% de los adul-

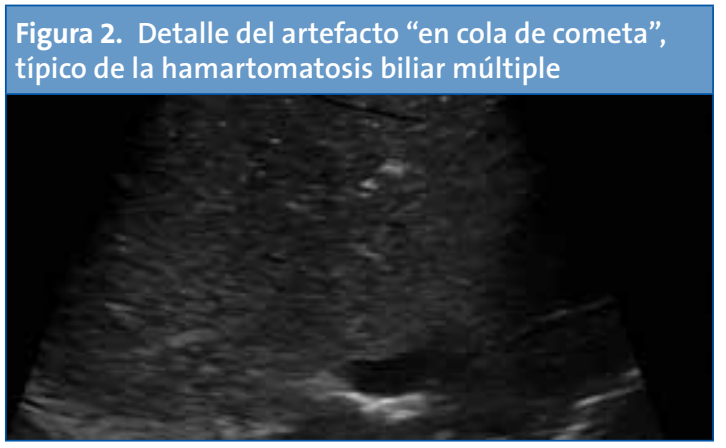

tos $^{1}$. Fue descrita por primera vez por von Meyenburg el año $1955^{2}$. También se conoce a esta lesión como microhamartomasis, adenomatosis de los conductillos biliares, colangioadenomatosis múltiple, conductos biliares intracapsulares aberrantes, fibroadenomatosis y hamartomatosis hepática. Su origen está en una disembriogénesis ${ }^{3}$. En nuestro conocimiento, no se ha vinculado etiológicamente hasta el momento la alergia a las proteínas de leche de vaca con la HBM, por lo que suponemos que la asociación con esta entidad ha sido un hecho casual.

Los hamartomas de la HBM tienen un color blanco-grisáceo, y pueden estar localizados en la región subcapsular o dispersos por el parénquima. Su tamaño suele ser inferior a $3 \mathrm{~mm}$, si bien en ocasiones pueden agruparse y alcanzar una longitud de $15 \mathrm{~mm}$ de diámetro ${ }^{3}$.

Con frecuencia se engloba a la HBM dentro del espectro de la enfermedad fibropoliquística del hígado, junto con las entidades que se muestran en la Tabla $1^{4}$. Desde el punto de vista de la localización, suele afectar de forma difusa a todo el hígado ${ }^{1-4}$.

Clínicamente, la HBM sigue un curso asintomático. Suele ser un hallazgo casual al realizar una prueba de imagen, al hacer una laparotomía o una autopsia. Si bien se considera que es una lesión benigna, de forma puntual se ha descrito su asociación con colangiocarcinoma. El carácter asintomático y el bajo riesgo de malignización marcan una diferencia esencial con respecto a la enfermedad de Caroli. Esta última cursa en forma de colangitis/pancreatitis recurrentes, y la tasa de malignización es del $7 \%^{5}$.

El diagnóstico de la HBM requiere inicialmente una prueba de imagen (ecografía, tomografía computarizada [TC] o resonancia magnética

Tabla 1. Espectro de la enfermedad fibropoliquística hepática

- Hamartomatosis biliar múltiple

- Enfermedad de Caroli

- Hamartomas mesenquimales

- Enfermedad poliquística hepática autosómica dominante

- Quiste de colédoco 
$[R M])^{1-6}$. En la ecografía, la presencia de hamartomas múltiples hace que el hígado muestre un aspecto granular, heterogéneo, en el que destaca la presencia de numerosas áreas redondeadas hipoanecoicas con zonas hipercogénicas, artefacto en "cola de cometa" y refuerzo posterior. Con el ecoDoppler (Fig. 3) se puede visualizar un artefacto "de centelleo" (alternancia de color inmediatamente detrás de un objeto ecogénico estacionario), debido a las reverberaciones producidas por los cristales de colesterol que rellenan en ocasiones los conductos dilatados $^{1-7}$. En la TC se aprecia la presencia de múltiples nódulos hipodensos ${ }^{8}$. Se ha postulado, especialmente en pacientes adultos, que la técnica de elección es la RM. Esta técnica aporta mayor sensibilidad y especificidad a la hora de hacer el diagnóstico diferencial con otros cuadros (Tabla 2). En la secuencia T1, los hamartomas se muestran como estructuras nodulares hipointensas con respecto al parénquima adyacente. En la secuencia T2 se ven como estructuras hiperintensas. Típicamente, los hamartomas no se comu-

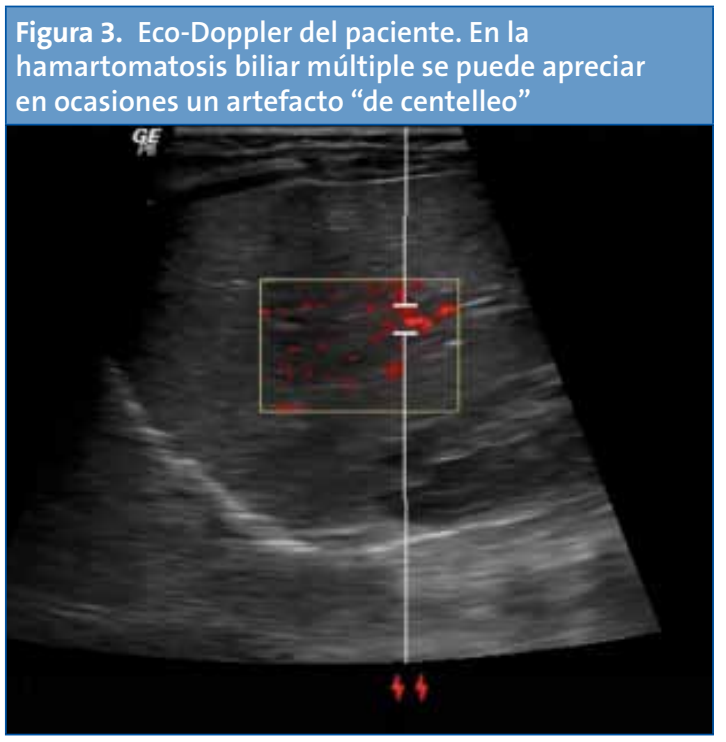

Tabla 2. Entidades incluidas en el diagnóstico diferencial con la hamartomatosis biliar múltiple

- Metástasis hepáticas múltiples

- Microabscesos

- Quistes

- Adenomas

- Hepatitis granulomatosa

- Cistoadenocarcinoma

nican con la vía biliar, al contrario de lo que ocurre en la enfermedad de Caroli.

El diagnóstico de certeza se hace a través de una biopsia hepática. Desde el punto de vista histológico, cada hamartoma consta de varios grupos de conductos biliares intrahepáticos dilatados en un estroma fibrilar de colágeno denso que puede hialinizarse y/o calcificarse. En el interior de los conductos dilatados puede hacer cristales de colesterol.

La HBM no precisa tratamiento, dado su bajo potencial de malignización. No hay establecido ningún protocolo de seguimiento, si bien nos parece recomendable la realización de una ecografía semestral o anual.

En resumen, la HBM es una lesión benigna, asintomática y frecuente en la edad pediátrica, que se advierte de manera casual al realizar una ecografía abdominal por otro motivo. No precisa tratamiento alguno. Recomendamos conducta expectante y seguimiento periódico en las consultas externas de Gastroenterología infantil.

\section{CONFLICTO DE INTERESES}

Los autores declaran no presentar conflictos de intereses en relación con la preparación y publicación de este artículo.

\section{ABREVIATURAS}

HBM: hamartomatosis biliar múltiple - RM: resonancia magnética $\bullet$ TC: tomografía computarizada. 


\section{BIBLIOGRAFÍA}

1. Tohme-Noun C, Cazals D, Noun R, Menassa L, Valla D, Vilgrain $\mathrm{V}$. Multiple biliary hamartomas: magnetic resonance features with histopathologic correlation. Eur Radiol. 2008;18:493-9.

2. Gil-Bello D, Ballesteros E, Sanfeliu E, Andreu FJ. Calcification in biliary hamartomatosis. Br J Radiol. 2012; 85:99-101.

3. Singhal A, Kanagala R, Wright HI, Kohli V. Multiple bile duct hamartomas mimicking diffuse hepatic metastasis: Gl image. J Gastrointest Surg. 2010;14: 1629-31.

4. Moon Shin Y. Biliary hamartoma presented as a single mass. Kor J Hepatol. 2011;17:331-4.
5. Bastart F, Vilá A, Padrós J, Escape I, Bruguera M. Multiple biliary hamartomatosis: a multidisciplinary diagnosis. Gastroenterol Hepatol. 1998;21:115.

6. Nagano Y, Matsuo K, Gorai K, Sugimori K, Kunisaki C, Ike $\mathrm{H}$, et al. Bile duct hamartomas (von Mayenburg complexes) mimicking liver metastases from bile duct cancer: MRC findings. World J Gastroenterol. 2006;12:1321-3.

7. Jeon SJ, Yoon SE. Color Doppler twinkling artifact in hepatic bile duct hamartomas (von Meyenburg complexes). J Ultrasound Med. 2006;25:399-402.

8. Mas A, Almirall J, Rodríguez A, Bella R, Corcuera A, Donoso L. Microhamartomatosis of the liver associated with autosomal dominant polycist kidney disease: CT and US appearance. J Comput Assist Tomagr. 1994;18:972-4. 